\title{
The Yersinia pestis gcvB gene encodes two small regulatory RNA molecules
}

\author{
Sarah D McArthur, Sarah C Pulvermacher and George V Stauffer*
}

Address: Department of Microbiology, The University of Iowa, Iowa City, Iowa 52242, USA

Email: Sarah D McArthur - sdmcarthur5@yahoo.com; Sarah C Pulvermacher - sarah-pulvermacher@uiowa.edu; George V Stauffer* - georgestauffer@uiowa.edu

* Corresponding author

Published: 12 June 2006

BMC Microbiology 2006, 6:52 doi:10.1 |86/|47|-2/80-6-52

This article is available from: http://www.biomedcentral.com/I47I-2/80/6/52

(C) 2006 McArthur et al; licensee BioMed Central Ltd.

This is an Open Access article distributed under the terms of the Creative Commons Attribution License (http://creativecommons.org/licenses/by/2.0), which permits unrestricted use, distribution, and reproduction in any medium, provided the original work is properly cited.
Received: 27 March 2006

Accepted: 12 June 2006

\begin{abstract}
Background: In recent years it has become clear that small non-coding RNAs function as regulatory elements in bacterial virulence and bacterial stress responses. We tested for the presence of the small non-coding GcvB RNAs in Y. pestis as possible regulators of gene expression in this organism.

Results: In this study, we report that the Yersinia pestis KIM6 gcvB gene encodes two small RNAs. Transcription of $g c v B$ is activated by the GcvA protein and repressed by the GcvR protein. The gcvB-encoded RNAs are required for repression of the $Y$. pestis $d p p A$ gene, encoding the periplasmic-binding protein component of the dipeptide transport system, showing that the GcvB RNAs have regulatory activity. A deletion of the gcvB gene from the $Y$. pestis KIM6 chromosome results in a decrease in the generation time of the organism as well as a change in colony morphology.

Conclusion: The results of this study indicate that the $Y$. pestis gcvB gene encodes two small noncoding regulatory RNAs that repress $d p p A$ expression. A gcvB deletion is pleiotropic, suggesting that the sRNAs are likely involved in controlling genes in addition to $d p p A$.
\end{abstract}

\section{Background}

Yersinia pestis is the causative agent of plague, an infectious disease that results in lymphatic and blood infections [1]. The $Y$. pestis genome has been sequenced $[2,3]$. $Y$. pestis carries three plasmids of approximately 9.5, 70, and 100 kilobasepairs and each carries genes necessary for or that contribute to the pathogenicity of the bacterium [1]. The 70 kilobasepair plasmid encodes the low-calcium response stimulon (LCRS). Components of the LCRS include Yops (secreted anti-host proteins) and a type III secretion apparatus, or Ysc. The type III secretion apparatus is responsible for the translocation of the Yops to host cells that in turn down-regulate the response of the host phagocytic cells to infection [4]. Natural LCRS-negative mutants of $Y$. pestis occur, resulting in avirulence of the bacteria [1]. Besides the three plasmids, another pathogenicity factor is pigmentation. Cells of $Y$. pestis adsorb hemin at $26^{\circ} \mathrm{C}$ but not at $37^{\circ} \mathrm{C}$ and are pigmented $\left(\mathrm{Pgm}^{+}\right)$and virulent. Spontaneous nonpigmented (Pgm-) mutants of $Y$. pestis have been isolated. The Yersiniabactin iron transport system is part of the pgm locus, and its loss results in a Pgm- mutant that is avirulent in mice unless hemin, ferrous sulfate, or ferric chloride is injected into mice along with the bacterial challenge [1]. 
Recently, a new class of molecules has been shown to regulate gene expression in bacteria, small non-coding regulatory RNAs (sRNAs). These sRNAs have gained much attention as recent genome-wide studies have identified sRNAs in a wide variety of organisms [5]. Most of these bacterial sRNAs are between 50 and 400 nucleotides (nts) in length and play important roles in global regulation $[6,7]$. Hfq is a small RNA binding protein and sRNAs in particular are targets for Hfq [6]. Binding of these sRNAs by Hfq in some way facilitates base pairing between the sRNAs and their respective target RNAs [8,9]. In Vibrio cholerae, sRNAs (Qrr RNAs) have been shown to regulate virulence genes [10] and in Brucella abortus an $h f q$ mutation is lethal [9]. These results suggest that sRNAs and Hfq likely play important roles in the virulence of certain Gram-negative pathogens.

The E. coli gcvB gene encodes sRNAs that are not translated in vivo [11]. A strain carrying a deletion of $g c v B$ has constitutive synthesis of OppA and DppA, periplasmic binding proteins of the two major peptide transport systems nor- mally repressed in cells grown in rich medium [12-14]. In addition to OppA and DppA, several other proteins were shown to increase or decrease in response to GcvB RNA levels, but the specific proteins were not identified [11]. Nevertheless, the results show that the GcvB RNAs are regulatory and possibly serve as global regulators. A computer search of the $Y$. pestis sequence showed that $Y$. pestis has a $g c v B$ gene that shares considerable sequence homology with the E. coli gcvB sequence (Fig. 1). Thus, the GcvB RNAs from $E$. coli likely have functional counterparts in $Y$. pestis. The results of this study show that the Y. pestis gcvB gene encodes two sRNAs that, in turn, have regulatory activity. In addition, a deletion of the gcvB gene from the $Y$. pestis chromosome alters growth rate and colony morphology.

\section{Results and discussion Identification of the Y. pestis gcvB gene}

The E. coli $g c v B$ gene is divergently transcribed from $g c v A$, which encodes the activator protein for $g c v B$ expression (Fig. 1) [11]. Thus, we used computer searches of genome

$$
\begin{aligned}
& \text { Ec gtaacctattagttttttaatctgagccattataattgtccgttgagct } \frac{-35}{-10}+\frac{-10}{-35}+1 \\
& \text { Yp tttacttattagttttataatccgagc cattataattgtccattgaggatacgccagtaatacctatagtggccgcact }
\end{aligned}
$$

Ec tcctg-agccggaacgaaaagttttatcggaatgcgtgtt-ctggtgaacttttggcttacggttgtgatgttgtgttgttg

Yp tcccctagc ggaacgaaaagtaggtttggtatcccaggtactgaaatgcttttggctt------- tgtggtggtgatgttg $\uparrow$ TF-1

Ec tgtttgcaattggtctgcgattcagaccatggtagcaaagctacc---ttttttcacttcctgtacatttaccetgtctgtc

Yp tgtttgcaaatggtctggtatgccagacatagtagctaagctactgctttttttcacttcctgtacatttaccctgtctgtc $\rightarrow \mathrm{t} 1$

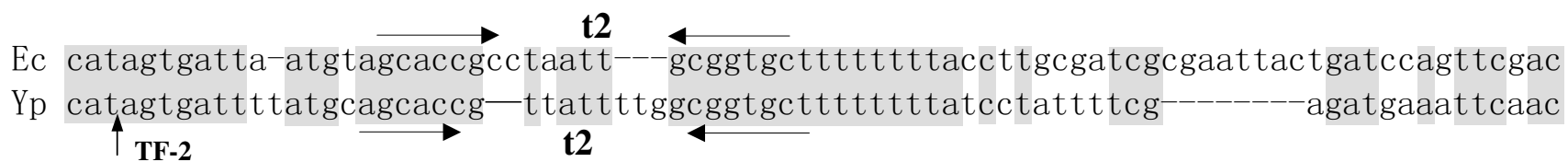

Ec catctcttc-acgtcagtacggt

Yp ttgttgttgctaccaccttaatgt

\section{Figure I}

Comparison of the $E$. coli and $Y$. pestis gcvA/gcvB control regions and gcvB genes. Ec, $E$. coli; Yp, Y. pestis. Bases that are identical are boxed in gray. The $E$. coli promoter -10 and -35 sequences are underlined for gcvA [29] and overlined for gcvB [ I I ]. Arrows indicate transcription start sites and directions of transcription of gcvA and gcvB. The GcvA binding region is indicated above the sequence [30]. The deduced $Y$. pestis -10 and -35 promoter sequences are underlined for gcvA and overlined for gcvB, and the deduced GcvA binding site is indicated above the sequence. Two Rho-independent transcription terminators for the $E$. coli

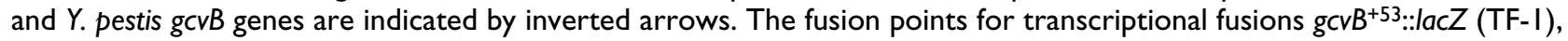
$g c v B^{+164:: l a c Z(T F-2)}$ and $g c v B^{+251:: l a c Z}(T F-3)$ are indicated by vertical arrows. 
sequences with the gcvA gene product as a query to predict $g c v B$ homologs in other organisms. We identified GcvBlike RNA sequences in the genera Yersinia, Salmonella, Haemophilus, Vibrio, Pasteurella, Shigella, Erwinia, Klebsiella, Photorhabdus and Actinobacillus. Despite considerable sequence variation in many of these homologs, they are predicted by the $m$ fold algorithm [15] to assume a similar secondary structure. A comparison of three of these GcvB RNAs is shown in Fig. 2. The location of the putative $Y$. pestis $g c v B$ gene adjacent to and divergent from $g c v A$, its $77 \%$ sequence similarity to the $E$. coli $g c v B$ sequence and its predicted secondary structure make it a likely homolog of $g c v B$ in $Y$. pestis. Furthermore, identical $g c v B$ sequences can be found in all other $Y$. pestis strains presently in the data base, both virulent and avirulent strains.

\section{The $Y$. pestis gcvB gene encodes two sRNAs}

The E. coli gcvB gene encodes two sRNA transcripts that are not translated in vivo [11]. To determine if the $g c v B$ gene in $Y$. pestis is functional and possibly encodes sRNAs, we initially constructed plasmid $\mathrm{p} g c v B^{\mathrm{Yp}+53}::$ lacZ, carrying a transcriptional fusion of the $g c v B$ gene at basepair (bp) +53 to lacZ. Plasmid pgcv $B^{\mathrm{Yp}+53}::$ lacZ and the vector alone were transformed into $Y$. pestis strain KIM6, the transformants grown in heart-infusion broth (HIB) + ampicillin (AP) to mid-log phase of growth and the cultures assayed for $\beta$-galactosidase activity. The KIM6 and KIM6 [pMC1403] control transformant gave $6 \pm 0.3$ and $6 \pm 1$ units of $\beta$-galactosidase activity, respectively, whereas the KIM6 [pgcvB ${ }^{\mathrm{Yp}+53:: l a c Z]}$ transformant gave 5,985 \pm 118 units of $\beta$-galactosidase activity. The results suggest that the $g c v B$ gene is expressed in $Y$. pestis.

The gcvB gene from $Y$. pestis possesses two possible Rhoindependent transcription terminators, which if functional, would allow the production of two sRNAs of about $130 \mathrm{nts}$ and $206 \mathrm{nts}$ (Fig. 1). Three transcriptional gene fusions of the $Y$. pestis $g c v B$ gene to lac $Z$ were created to determine if these putative terminator sites function as transcription terminators in vivo. The three fusions, designated $\quad \lambda g c v B^{\mathrm{Yp}+53}:: l a c Z, \quad \lambda g c v B^{\mathrm{Yp}+164:: l a c Z}$ and

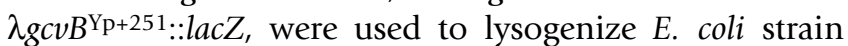
GS162. The lysogens were then grown in Luria-Bertani broth (LB) [16] to mid-log phase of growth and the cultures assayed for $\beta$-galactosidase activity. About $44 \%$ of the $\beta$-galactosidase activity seen when the fusion precedes

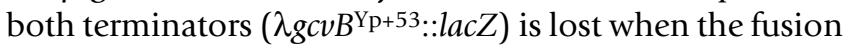

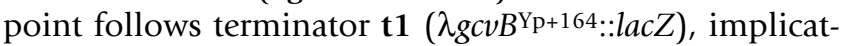
ing $\mathbf{t} 1$ as a site of transcription termination in vivo (Table 1 ). The remaining activity that escapes termination by $\mathbf{t} 1$

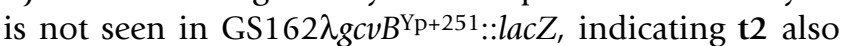
functions as a terminator in vivo (Table 1). When the 206 nts preceding terminator $\mathbf{t} 2$ for $g c v B$ were analyzed, there were only short open reading frames (ORFs) that could encode polypeptides of 36 amino acids or less. These ORFs all lack good translational start sites and were not tested to determine if they encode small polypeptides. The E. coli GcvB RNAs are not translated into polypeptides [11]. Thus, we conclude that the products of the $g c v B$ gene in $Y$. pestis are two sRNAs that are not translated, although

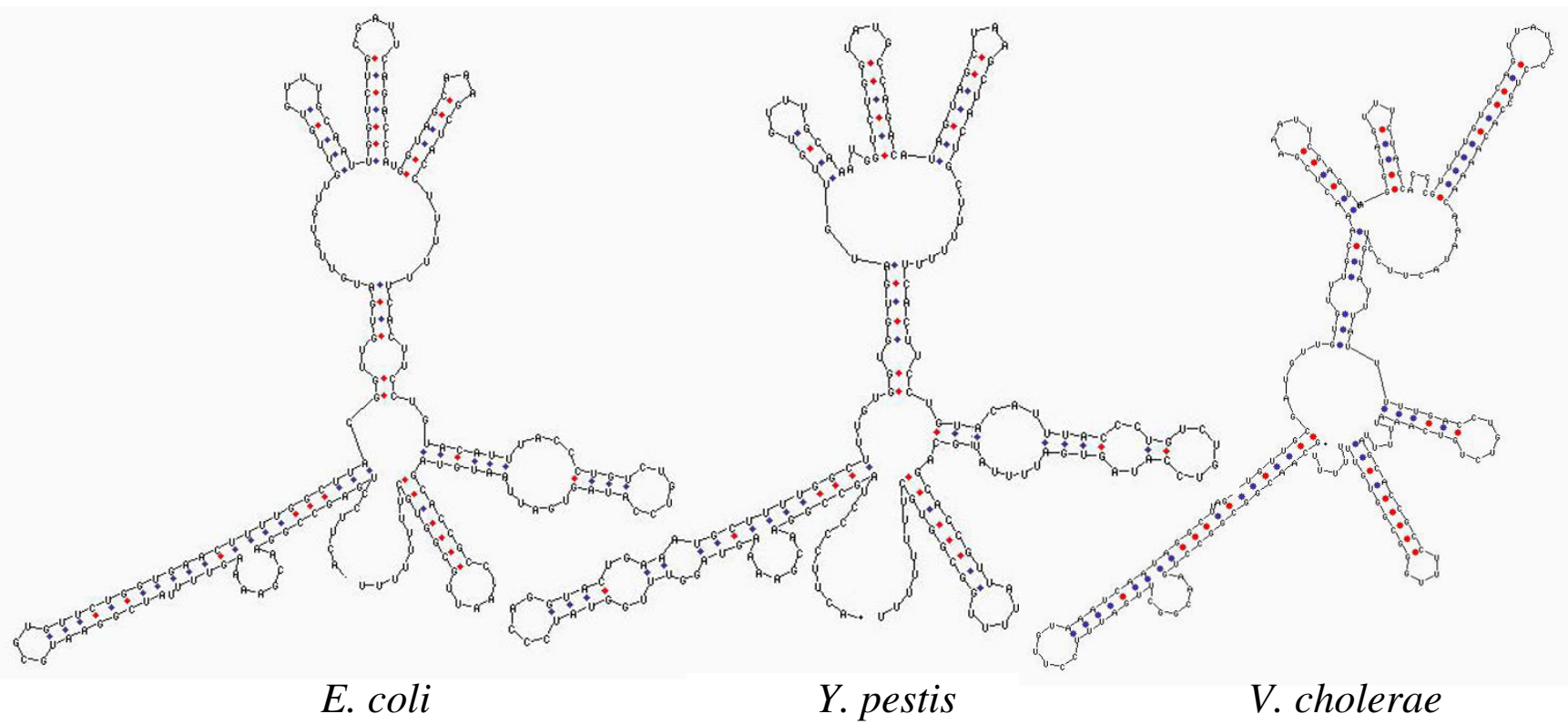

\section{Figure 2}

Secondary structures of GcvB RNAs with 77\% (Y. pestis) and 53\% (V. cholerae) identity to the E. coli GcvB RNA as predicted by the mfold algorithm [15]. 
Table I: The $Y$. pestis gcvB gene encodes two sRNAs. Cells were grown in LB to an $\mathrm{OD}_{600}$ of $\sim 0.5$ and assayed for $\beta$-galactosidase activity [16]. Activity is expressed in Miller units.

\begin{tabular}{|c|c|c|}
\hline Transformant & Relevant genotype & $\beta$-Galactosidase activity \\
\hline GSI62 & WT & $1 \pm 0.1$ \\
\hline GSI $62 \lambda g c v B Y p+53:: l a c Z$ & WT & $369 \pm 40$ \\
\hline GSI $62 \lambda$ gcvBYp+164::IacZ & WT & $182 \pm 7$ \\
\hline GSI $62 \lambda g c v B{ }^{Y_{p}+251}::: \operatorname{lacZ}$ & WT & $\mathrm{I} \pm 0.3$ \\
\hline
\end{tabular}

the results do not completely rule out the possibility that the Y. pestis GcvB sRNAs encode small peptides. In E. coli, about $90 \%$ of the transcripts that initiate at the $g c v B$ promoter terminate at terminator $\mathbf{t} 1$, and the remaining $10 \%$ terminate at terminator $\mathbf{t} 2$ [11]. A comparison of the $E$. coli and $Y$. pestis $\mathbf{t} 1$ sites shows that an additional 2 bps occur between the predicted GC-rich stem-loop structure and the run of $T$ residues in the $Y$. pestis $\mathbf{t} 1$ site that are not present in the E. coli sequence, suggesting that the $Y$. pestis t1 site is likely less functional as a transcription terminator than the E. coli t1 site (Fig. 1). Nevertheless, the results are consistent with the $Y$. pestis $g c v B$ gene encoding two sRNA molecules of about 130 and 206 nts and in roughly equal amounts.

We used Northern blotting to confirm that the gcvB locus in Y. pestis encodes sRNA transcripts of about 130 and 206 nts. Two small RNA molecules were detected in RNA isolated from $Y$. pestis KIM6 grown in HIB medium using a probe specific for the gcvB locus (Fig. 3). These results are consistent with the in vivo results with the $Y$. pestis gcvB transcriptional fusions.

\section{Regulation of the $\mathbf{Y}$. pestis gcvB gene}

The E. coli gcvB gene is activated by GcvA in the presence of glycine and repressed by GcvA + GcvR in its absence; this repression is enhanced by the addition of purines [11]. The regulation of the $Y$. pestis gcvB gene was tested with respect to the effects of glycine and purine supplementation to the growth medium and with respect to the GcvR and GcvA proteins and the GcvB RNAs. For these experiments we used the $\lambda g c v B^{\mathrm{Y} p+53:: l a c Z}$ fusion to lysogenize appropriate $E$. coli host strains. The lysogens were grown in glucose minimal (GM) or GM supplemented with glycine or inosine to mid-log phase of growth and assayed for $\beta$-galactosidase levels. In the wild-type (WT) GS162 $\lambda g c v B^{\mathrm{Y}} \mathrm{p}+53::$ lac $Z$ lysogen, the addition of glycine to GM growth medium resulted in an 11.5-fold induction of $\beta$-galactosidase expression, whereas the addition of the purine inosine resulted in a 2.5 -fold repression below the unsupplemented GM level (Table 2, line 1). In the gcvA

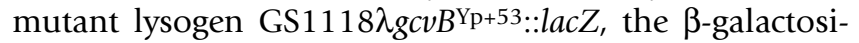
dase levels were low and non-inducible by glycine (Table 2 , line 2). The addition of inosine had no significant effect in the $g c v A$ mutant strain. In the $g c v R$ mutant lysogen

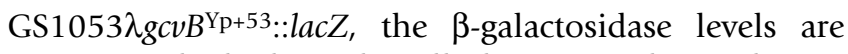
constitutively high under all three growth conditions (Table 2, line 3 ). The results suggest that activation of the $Y$. pestis $g c v B$ gene requires the GcvA protein and that repression requires the GcvR protein. Whether the negative regulation by GcvR requires a direct interaction of GcvR with GcvA as in E. coli $[17,18]$ awaits further investigation. Furthermore, there appears to be no autoregulation of $g c v B$ by its own sRNA products as the $g c v B$ mutant lysogen GS1144 $\lambda g c v B^{\mathrm{Yp}+53}::$ lac $Z$ shows normal regulation of the $g c v B^{\mathrm{Y} p+53}::$ lacZ fusion (Table 2, line 4).

\section{Y. pestis gcvA encodes an activator protein for gcvB expression}

Since activation of the $Y$. pestis $g c v B^{\mathrm{Yp}+53}$ fusion in E. coli was dependent on GcvA (Table 2), we determined if the $Y$. pestis gcvA gene also encodes an activator protein for $g c v B$ expression. We assumed this would be the case, as the $E$. coli and Y. pestis GcvA proteins are $88 \%$ identical at the

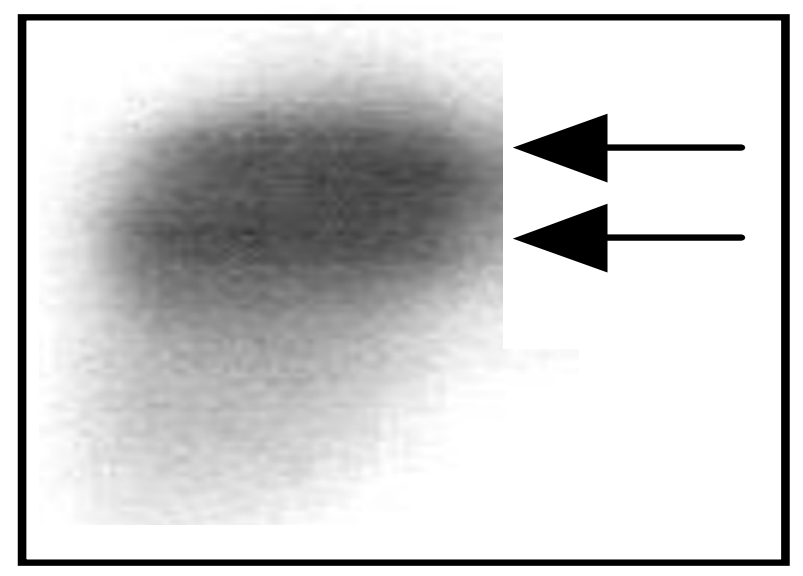

\section{Figure 3}

Northern blot analysis of GcvB from Y. pestis strain KIM6. Total cell RNA was isolated from strain KIM6 grown in HIB at $30^{\circ} \mathrm{C}$ to an O.D. 600 of 0.7 and probed with a ${ }^{32}$ P-labeled GcvB specific DNA probe as described in Methods. Two gcvB transcripts of about 206 and 130 nucleotides identified are indicated with arrows. Their sizes were determined based on their mobilities relative to the mobility of the $E$. coli GcvB RNA and 5S rRNAs (not shown). 
Table 2: Regulation of the $Y$. pestis gcvB ${ }^{+53}$ ::lacZ transcriptional fusion in E. coli. Cells were grown in GM media with the indicated supplements to an $O D_{600}$ of $\sim 0.5$ and assayed for $\beta$-galactosidase activity [16]. Activity is expressed in Miller units.

\begin{tabular}{|c|c|c|c|c|}
\hline \multirow[t]{2}{*}{ Lysogen } & \multirow[t]{2}{*}{ Relevant genotype } & \multicolumn{3}{|c|}{$\beta$-galactosidase activity for cells grown in: } \\
\hline & & GM & GM + glycine & GM + inosine \\
\hline GSI $62 \lambda g c v B Y_{p}+53:: / a c Z$ & WT & $15 \pm 2$ & $173 \pm 2$ & $6 \pm 3$ \\
\hline GSIII $8 \lambda g c v B Y_{p}+53:: / a c Z$ & $\Delta g c v A$ & $2 \pm 1$ & $3 \pm 1$ & $2 \pm 1$ \\
\hline 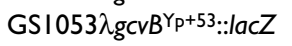 & $g c v R$ & $620 \pm 58$ & $419 \pm 13$ & $440 \pm 174$ \\
\hline GSII $44 \lambda g c v B Y_{p}+53:: / a c Z$ & $\Delta g c v B$ & $10 \pm 2$ & $140 \pm 24$ & $6 \pm 2$ \\
\hline
\end{tabular}

amino acid sequence level. The $Y$. pestis gcvA gene was cloned into plasmid pACYC177 and tested for its ability to complement an E. coli gcvA mutant. The E. coli strain GS1132 carries a deletion of the gcvA gene [11]. This strain was lysogenized with an E. coli $\lambda g c v B$ ::lacZ transcriptional gene fusion and subsequently transformed with the control plasmid pACYC177, or pACYC177 carrying either the E. coli or the Y. pestis gcvA gene. The cells were grown in LB to mid-log phase of growth and assayed for $\beta$-galactosidase activity. As reported previously [11], expression of the E. coli gcvB::lacZ fusion was increased about 400 -fold in the presence of the E. coli gcvA gene (Table 3, line 3). The $Y$. pestis gcvA gene also complemented the E. coli $\triangle g c v A$ strain, restoring $g c v B:: l a c Z$ expression to nearly the same level as seen with the E. coli gcvA gene (Table 3, line 4). These results show that the $Y$. pestis gcvA gene codes for an activator protein capable of activating expression of an E. coli gcvB::lacZ fusion.

\section{The $Y$. pestis gcvR gene encodes a repressor protein for gcvB expression}

Since deletion of the $g c v R$ gene in $E$. coli results in constitutive expression of the $Y$. pestis $g c v B^{\mathrm{Yp}+53}$ fusion (Table 2), we tested if the $Y$. pestis gcvR gene encodes a repressor for $g c v B$ expression. We assumed this would be the case, as the E. coli and Y. pestis GcvR proteins are 75\% identical at the amino acid sequence level. The E. coli strain GS1053 carries a Tn10 element inserted into the $g c v R$ gene [19]. This strain was lysogenized with an E. coli $\lambda g c v B:: l a c Z^{+50}$ transcriptional gene fusion [11] and subsequently transformed with the control plasmid pACYC177, or pACYC177 carrying either the E. coli gcvR gene or the $Y$. pestis gcvR gene. The cells were grown in GM media to mid-log phase of growth and assayed for $\beta$-galactosidase activity. Expression of the E. coli gcvB::lacZ fusion is constitutive in the absence of a functional GcvR protein (Table 4, lines 1 and 2). The gcvB::lacZ fusion, however, was repressed in the presence of either pGS601, carrying E. coli $g c v R$, or $\mathrm{p} g c v R^{\mathrm{Yp}-\mathrm{p} 177}$, carrying Y. pestis $g c v \mathrm{R}$ (Table 4, lines 3 and 4).

In E. coli, the GcvA and GcvR proteins interact to form a repressor complex $[17,18]$. The above results suggest that the Y. pestis GcvR protein interacts with the E. coli GcvA protein to form a repression complex. We tested if the $Y$. pestis $g c v A$ and $g c v R$ gene products also likely form a repressor complex to control expression of an E. coli $g c v B::$ lacZ fusion. Strain GS1131 $\lambda g c v B:: l a c Z$ carries $\Delta g c v R$ $\triangle g c v A$ mutations. Strain GS1131 $\lambda g c v B::$ lacZ was transformed with plasmid $\mathrm{p} g c v A^{\mathrm{Yp}-\mathrm{p} 177, \mathrm{p} g c v R^{\mathrm{Yp}} \mathrm{p} 322 \text {, or both }}$ plasmids. The vectors for $\mathrm{p} g c v A^{\mathrm{Yp}-\mathrm{p} 177}$ and $\mathrm{p} g c v R^{\mathrm{Yp}-\mathrm{p} 322}$ are pACYC177 and pBR322, respectively, to insure an excess of $\mathrm{GcvR}^{\mathrm{Yp}}$ versus $\mathrm{GcvA}^{\mathrm{Yp}}$. The cells were grown in GM media + appropriate antibiotics, harvested in mid-log phase of growth and assayed for $\beta$-galactosidase activity. The $Y$. pestis gcvA gene complemented the $\Delta g c v A$ mutation, resulting in activation of the $g c v B:: l a c Z$ fusion (Table 4, line 7). The $Y$. pestis gcvR gene complemented the $g c v R$ mutation, as repression of the $g c v B::$ lac $Z$ fusion occurred in the $\mathrm{p} g c v A^{\mathrm{Yp}-\mathrm{p} 177} \mathrm{p} g c v R^{\mathrm{Yp}-\mathrm{p} 322}$ double transformant (Table 4, line 8). These results suggest that the GcvA and GcvR proteins likely interact to form a repression complex in $Y$. pestis. In E. coli, GcvA also activates the gcvTHP operon and GcvA + GcvR repress the operon $[17,18]$.

Table 3: The $Y$. pestis gcvA gene encodes an activator protein. Cells were grown in LB to an $O D_{600}$ of $\sim 0.5$ and assayed for $\beta-$ galactosidase activity [16]. Activity is expressed in Miller units.

\begin{tabular}{|c|c|c|}
\hline Lysogen & Relevant genotype & $\beta$-Galactosidase activity \\
\hline 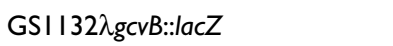 & $\Delta(g c v A$ gcvB $)$ & $<1$ \\
\hline 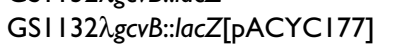 & $\Delta(g \subset v A$ gcvB $)$ & $<1$ \\
\hline 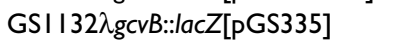 & $\Delta(g c v A$ gcvB $) / g c v A$ Ec & $399 \pm 22$ \\
\hline GSII $32 \lambda$ gcvB::IacZ[pgcvAYp-p177] & $\Delta(g c v A g c v B) / g c v A^{Y p}$ & $254 \pm 21$ \\
\hline
\end{tabular}


Table 4: The $Y$. pestis gcvR gene complements an $E$. coli gcvR mutation. Cells were grown in $G M$ media to an $O D_{600}$ of $\sim 0.5$ and assayed for $\beta$-galactosidase activity [16]. Activity is expressed in Miller units.

\begin{tabular}{|c|c|c|}
\hline Lysogen & Relevant genotype & $\beta$-Galactosidase activity \\
\hline 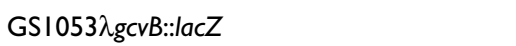 & GcvR & $308 \pm 19$ \\
\hline 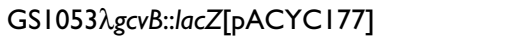 & GcvR & $384 \pm 175$ \\
\hline 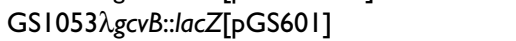 & $g c v R / g c v R E c$ & $11 \pm 1.5$ \\
\hline 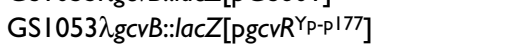 & $g c v R / g c v R Y_{p}$ & $14 \pm 1.2$ \\
\hline 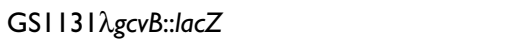 & $\Delta g c v A \Delta g c v R$ & $2.2 \pm 0.2$ \\
\hline GSI| $3 \mid \lambda g c v B:: / a c Z\left[p g c v R^{Y p-p 322}\right]$ & $\Delta g c v A \Delta g c v R / g c v R^{Y p}$ & $2.8 \pm 0.1$ \\
\hline GSI| $3|\lambda g c v B::| a c Z\left[p g c v A^{Y p-p \mid 77]}\right.$ & $\Delta g c v A \Delta g c v R / g c v A^{Y} \mathrm{P}$ & $393 \pm 8$ \\
\hline GSI I 3 I $\lambda g c v B:: l a c Z\left[p g c v A^{Y p-177} p g c v R^{Y p-p 322}\right]$ & $\Delta g c v A \Delta g c v R / g c v A^{Y p} g c v R^{Y p}$ & $6.8 \pm 0.4$ \\
\hline
\end{tabular}

Whether the Y. pestis GcvA and GcvR proteins also regulate the $Y$. pestis gcvTHP operon, or have additional regulatory roles, awaits further investigation.

The $Y$. pestis GcvB RNAs regulate the E. coli and $Y$. pestis dppA genes

The E. coli $g c v B$ gene negatively regulates the $d p p A$ and oppA genes [11]. In addition, many other genes were shown to be either negatively or positively regulated by the GcvB RNAs [11]. Thus, the E. coli GcvB RNAs are likely global regulators of gene expression. Y. pestis has homologs of $d p p A$ and $o p p A$. To determine if the $Y$. pestis GcvB RNAs are regulatory, we transformed an E. coli $\Delta$ $g c v B \lambda d p p A:: l a c Z$ lysogen with $\mathrm{p} g c v B^{\mathrm{Yp}-\mathrm{p} 322}$, the transformant and the parent lysogen were grown in LB to mid-log phase of growth and assayed for $\beta$-galactosidase levels. As expected, deletion of $g c v B$ caused an increase in $d p p A:: l a c Z$ expression (Table 5, line 2). However, pgcvB $\mathrm{Yp}$-p322 complemented the E. coli $\Delta g c v B$ mutation, repressing the $E$. coli $d p p A:$ :lacZ fusion (Table 5, line 3). Thus, the $Y$. pestis GcvB RNAs regulate the E. coli $d p p A:$ :lacZ fusion. We then tested the regulatory activity of the GcvB RNAs in $Y$. pestis directly. A single-copy plasmid carrying a $Y$. pestis $d p p A:: l a c Z$ fusion was used to transform $Y$. pestis strain KIM6 and KIM $6 \Delta g c v B$. The transformants were grown in $\mathrm{HIB}+\mathrm{AP}$ to mid-log phase of growth and assayed for $\beta$ galactosidase levels. Deletion of the $g c v B$ gene resulted in a 7.3-fold increase in $d p p A::$ lacZ expression (Table 5, compare lines 4 and 5). The results suggest that the $Y$. pestis GcvB RNAs are regulatory molecules. However, the mech- anism of GcvB RNA repression of $d p p A$ has not been determined. Although there is a region of 13-14 nucleotides in the $Y$. pestis GcvB RNA that can potentially base-pair with both the E. coli and $Y$. pestis $d p p A$ mRNAs near their ribosome binding sites, further studies are necessary to determine if base-pairing of GcvB RNA and $d p p A$ mRNA is part of the regulatory mechanism. Furthermore, in E. coli, the 206 nucleotide GcvB RNA is required for repression of $o p p A$ and $d p p A$ [11]. We are constructing a plasmid that will only produce the 130 nucleotide $Y$. pestis GcvB RNA to determine whether the 130 or 206 nucleotide RNA species is required for activity in $Y$. pestis.

\section{Deletion of the $Y$. pestis gcvB gene slows growth rate and alters colony morphology}

The KIM $6 \Delta g c v B$ strain routinely gave smaller colonies on HIB plates than the parent KIM6 strain. Thus, we investigated the growth of KIM $6 \Delta g c v B$ to determine the effect of the $\Delta g c v B$ mutation on growth rate. The parent strain KIM6, KIM6 $\Delta g c v B$ and KIM6 $\Delta g c v B\left[\mathrm{pgcv} B^{\mathrm{Yp}-\mathrm{sc}}\right]$ were grown in HIB broth at $37^{\circ} \mathrm{C}$. The generation times were then calculated. The KIM6 generation time at $37^{\circ} \mathrm{C}$ was $135 \pm 15$ minutes whereas KIM $6 \Delta g c v B$ had a generation time of 194 \pm 20 minutes (Fig. 4). The presence of $\mathrm{pgcvB} \mathrm{Bp}^{\mathrm{Yp}-\mathrm{sc}}$ in KIM $6 \Delta g c v B$ complemented the $g c v B$ deletion, as the generation time was reduced to $155 \pm 8$ minutes, close to the generation time of strain KIM6. Thus, deletion of the $g c v B$ gene impairs the ability of $Y$. pestis to grow as well as the parent strain on either solid media or in liquid media. This is in contrast to E. coli gcvB deletion mutants that

Table 5: Regulation of $E$. coli and $Y$. pestis $d p p A:: l a c Z$ translational gene fusions by the $Y$. pestis gcvB gene. Cells were grown in LB (E. coli) or in HIB (Y. pestis) at $37^{\circ} \mathrm{C}$ to an $\mathrm{OD}_{600} \sim 0.5$ and assayed for $\beta$-galactosidase activity [16]. Activity is expressed in Miller units. The parent strains $\mathrm{KIMG}$ and $\mathrm{KIM} 6 \triangle \mathrm{gcvB}$ grown in $\mathrm{HIB}$ at $37^{\circ} \mathrm{C}$ showed $<5$ units of $\beta$-galactosidase activity.

\begin{tabular}{|c|c|c|}
\hline Lysogen & Relevant genotype & $\beta$-Galactosidase activity \\
\hline 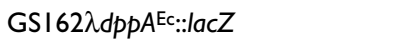 & WT & $103 \pm 24$ \\
\hline 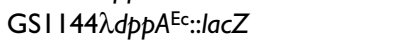 & $\Delta g c v B$ & $554 \pm 81$ \\
\hline 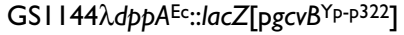 & $\Delta g c v B / g c v B Y_{P}$ & $154 \pm 32$ \\
\hline KIM6[pdppAYР::IacZ] & WT & $62 \pm 13$ \\
\hline $\mathrm{KIM} 6 \Delta g c v B\left[\mathrm{pdpp} A^{Y \mathrm{P}:: / a c Z]}\right.$ & $\Delta g c v B$ & $455 \pm 7$ \\
\hline
\end{tabular}




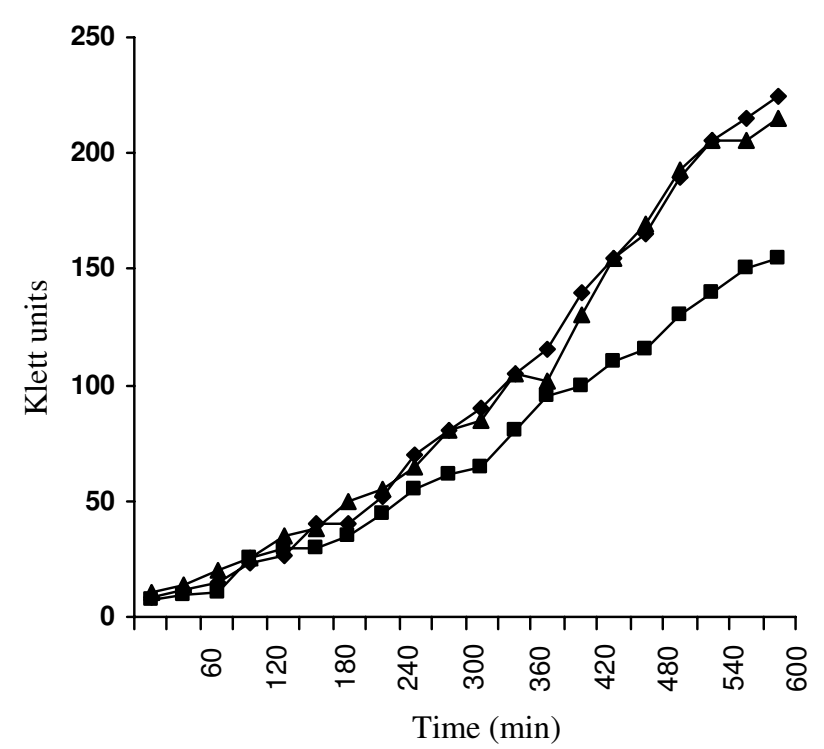

\section{Figure 4}

Effects of the $\triangle g c v B$ mutation on $Y$. pestis growth rates. $Y$. pestis strains $\mathrm{KIM} 6(\diamond), \mathrm{KIM} 6 \Delta g c v B(\square)$, and $\mathrm{KIM} 6 \Delta g c v B\left[p g c v B \mathrm{Y}^{\mathrm{p}}\right.$ sc] $(\boldsymbol{A})$ were grown in HIB (+ AP for the pgcvBYP-sc transformant) at $37^{\circ} \mathrm{C}$. The experiment was repeated three times. The curves show the results of a representative experiment.

have no observable phenotype. The KIM6 $\operatorname{gcvB}$ strain also showed a different colony morphology from WT KIM6. WT KIM6 colonies appear smooth and sticky, whereas the KIM $6 \Delta$ gcvB colonies appear dry and compact. The presence of $\mathrm{p} g c v B^{\mathrm{Yp}-\mathrm{sc}}$ in KIM $6 \Delta g c v B$ again complemented the $g c v B$ deletion, as the phenotype was restored back to the WT colony morphology.

In E. coli, many genes respond to the GcvB RNAs [11]. The pleiotropic nature of the $Y$. pestis $g c v B$ deletion suggests that the Y. pestis GcvB RNAs are likely global regulators as well. Identification of the specific genes regulated by the GcvB RNAs that are responsible for the altered phenotype will allow us to test directly their involvement in virulence of the organism. In addition, the GcvB sequences and regulatory regions from bp -90 to +1 , which include the putative GcvA binding sites for activation of $g c v B$, are $100 \%$ identical in all Yersina pestis strains, and greater than $92 \%$ identical in other Yersinia species. Thus, expression of $g c v B$ and the regulatory mechanisms of the GcvB RNAs are likely similar in all Yersinia species.

\section{Conclusion}

In summary, the $Y$. pestis gcvB gene is activated by the GcvA protein and repressed by the GcvR protein. The $g c v B$ gene encodes two sRNAs that have regulatory activity, repressing $d p p A$ expression. A gcvB deletion is pleiotropic, suggesting that the GcvB RNAs possibly serve as global regulators in $Y$. pestis.

\section{Methods \\ Bacterial strains, plasmids and phage}

Bacterial strains, plasmids and phage used in this study are listed in Table 6 or are described in the text.

\section{Media}

For E. coli strains, the complex medium used was LB [16] and the defined medium used was the minimal salts of Vogel and Bonner [20] supplemented with $0.4 \%$ glucose. GM media was always supplemented with $50 \mu \mathrm{g} \mathrm{ml}^{-1}$ of phenylalanine and $1 \mu \mathrm{g} \mathrm{ml}^{-1}$ of vitamin B1, since all E. coli strains carry pheA, thi mutations. Where indicated, glycine and inosine were added at $300 \mu \mathrm{g} \mathrm{ml}^{-1}$ and $50 \mu \mathrm{g} \mathrm{ml}^{-1}$, respectively. For $Y$. pestis strains, HIB was used [21]. Agar was added at $1.5 \%$ to make solid media. Antibiotics were added at the following concentrations: $\mathrm{AP}, 150 \mu \mathrm{g} \mathrm{ml}^{-1}$ for multi-copy plasmids and $50 \mu \mathrm{g} \mathrm{ml}^{-1}$ for single-copy plas-

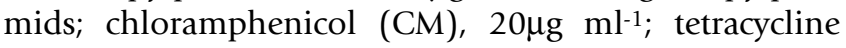
(TC), $10 \mu \mathrm{g} \mathrm{ml}^{-1}$.

\section{$\beta$-galactosidase assays}

$\beta$-galactosidase assays were performed on mid-log phase cells $\left(\mathrm{OD}_{600} \sim 0.5\right)$ as described by Miller [16]. Each experiment was repeated at least twice, with each sample assayed in triplicate.

\section{DNA manipulation}

Plasmid DNA was isolated using Qiagen Miniprep kits as described by the manufacturer (Qiagen). Restriction enzyme digestions and DNA ligations were carried out according to the manufacturer (New England Biolabs). DNA sequencing was performed by the University of Iowa DNA Core Facility.

\section{PCR}

PCR reactions were performed in $100 \mu$ l volumes. Each reaction mixture contained $10 \mu \mathrm{l} 10 \times$ polymerase buffer, $10 \mu \mathrm{l} 10 \times \mathrm{dNTPs}(0.2 \mathrm{mM}$ each $), 5 \mu \mathrm{l}$ Y. pestis DNA ( 15 $\mathrm{ng}), 100$ pmoles of forward and reverse primers designed specifically for each reaction, $1 \mu$ l of vent polymerase, and sterile water to bring the volume to $100 \mu \mathrm{l}$. PCR reactions were carried out under the following conditions: $5 \mathrm{~min}$ pre-incubation at $95^{\circ} \mathrm{C}$, and then 30 cycles of $95^{\circ} \mathrm{C}$ for 30 $\mathrm{sec}, 45^{\circ} \mathrm{C}$ for $30 \mathrm{sec}$, and $72^{\circ} \mathrm{C}$ for $2 \mathrm{~min}$.

\section{RNA extraction and Northern blot analysis}

Y. pestis KIM6 was grown in HIB at $30^{\circ} \mathrm{C}$ to an O.D. 600 of 0.7 , the cells collected for 1 minute in a microcentrifuge and immediately frozen at $-70^{\circ} \mathrm{C}$. Total cellular RNA was isolated using the MasterPure ${ }^{\mathrm{TM}}$ RNA purification kit (Epicenter). The final RNA pellet was re-suspended in water treated with diethyl pyrocarbonate and kept at $-70^{\circ} \mathrm{C}$. The RNA concentration was measured with a spectrophotometer at $260 \mathrm{~nm}$. RNA (10 $\mu \mathrm{g})$ was separated through a $1.5 \%$ formaldehyde gel and blotted on to a Biodyne Plus 
Table 6: Bacterial strains, plasmids and phage. All E. coli strains listed also carry $\Delta(\operatorname{argF-lac}) U I 69$, pheA905, thi, araDI29, rpsLI50, relAI, deoCl, flb530I, ptsF25 and rpsR mutations.

\begin{tabular}{|c|c|c|}
\hline Strains/plasmids/phage & Relevant genotype & Source/reference \\
\hline \multicolumn{3}{|l|}{ Strains* } \\
\hline GSI62 & WT & This laboratory \\
\hline GSI053 & $g c v R:: \operatorname{Tn} 10$ & [19] \\
\hline GSIII 8 & $\Delta g c v A: \Sigma a a d A$ & This laboratory \\
\hline GSII3I & $\Delta g c v A: \sum a a d A \Delta g c v R: \Sigma K N R$ & {$[\mathrm{II}]$} \\
\hline GSII32 & $\Delta(\operatorname{gcv} A$ gcvB $): \Sigma a a d A$ & {$[11]$} \\
\hline GSII44 & $\Delta g c v B: \Sigma C M^{R}$ & This laboratory \\
\hline KIM6 & $\mathrm{Icr}$ & {$[31]$} \\
\hline $\mathrm{KIM} 6 \Delta g c v B$ & $\Delta g c v B: \Sigma C M^{R}$ & This study \\
\hline \multicolumn{3}{|l|}{ Plasmid } \\
\hline pGS366 & Single-copy translational lacZ fusion vector & This laboratory \\
\hline pgcvB ${ }^{Y p-p 322}$ & Carries $Y$. pestis gcvB in pBR322 & This study \\
\hline PgcvB ${ }^{Y p-s c}$ & Carries $Y$. pestis gcvB in a single-copy vector & This study \\
\hline pgcvAYp-pI77 & Carries Y. pestis gcvA in pACYCI77 & This study \\
\hline $\mathrm{pgcv} R^{Y \mathrm{p}-\mathrm{p} 177}$ & Carries $Y$. pestis gcvR in pACYCI77 & This study \\
\hline $\mathrm{Pg} c v R^{\mathrm{YP}-\mathrm{p} 322}$ & Carries Y. pestis gcvR in pBR322 & This study \\
\hline$p d p p A^{Y} \mathrm{P}:: l a c Z$ & Y. pestis dppA::IacZ fusion in pGS366 & This study \\
\hline pGS335 & Carries E. coli gcvA in pACYCI77 & This lab \\
\hline pGS60I & Carries E. coli gcvR in $\mathrm{pACYCI} 77$ & This lab \\
\hline \multicolumn{3}{|c|}{ 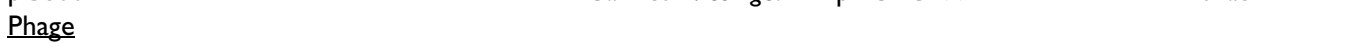 } \\
\hline$\overline{\lambda d p p A:: l a c Z}$ & $\lambda$ gt2 with $E$. coli $d p p A:$ :IacZ translational fusion & {$[\mathrm{II}]$} \\
\hline$\lambda g c v B:: l a c Z$ & $\begin{array}{l}\lambda \text { gt2 with } E \text {. coli gcvB }+50:: l a c Z \text { transcriptional } \\
\text { fusion }\end{array}$ & {$[11]$} \\
\hline$\lambda g c v B^{Y p}+53:: / a c Z$ & 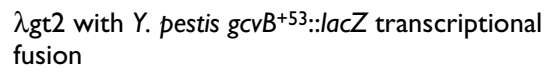 & This study \\
\hline$\lambda g c v B Y_{p}+164:: 1 a c Z$ & $\begin{array}{l}\lambda g t 2 \text { with } Y \text {. pestis gcvB }+164: \text { :IacZ transcriptional } \\
\text { fusion }\end{array}$ & This study \\
\hline$\lambda g c v B^{Y} p+251:: / a c Z$ & $\begin{array}{l}\lambda \text { gt2 with } Y \text {. pestis gcvB }{ }^{+251}: \text { :lacZ transcriptional } \\
\text { fusion }\end{array}$ & This study \\
\hline
\end{tabular}

Membrane (ISC BioExpress). The blot was hybridized with a PCR generated DNA fragment from bp +1 to +198 of the $Y$. pestis gcvB gene and ${ }^{32}$ P-labeled using the RediprimeTM II Random Prime Labeling System (Amersham Biosciences). Hybridization of the blot was at $58^{\circ} \mathrm{C}$ as described [22].

\section{Construction of gcvA, gcvB and gcvR plasmids}

The $Y$. pestis gcvB gene was cloned as follows. PCR primer YP-GCVB1F has an artificial EcoRI site and is complementary to the $Y$. pestis KIM6 DNA sequence beginning 114 bases upstream of the $g c v B$ transcription start site. PCR primer YP-GCVB2R has an artificial HindIII site and is complementary to the $Y$. pestis DNA sequence beginning 45 bases downstream of the $g c v B$ transcriptional termination site $\mathrm{t} 2$ (Fig. 1). Following PCR amplification, using $Y$. pestis chromosomal DNA as template, the amplified DNA was digested with EcoRI and HindIII, the $400 \mathrm{bp}$ fragment carrying $g c v B$ isolated from a $1 \%$ agarose gel and ligated into the EcoRI and HindIII sites of plasmid pBR322 [23], generating plasmid $\mathrm{p} g c v B^{\mathrm{Yp}-\mathrm{p} 322}$. The $Y$. pestis $g c v A$ and $g c v R$ genes were cloned using a similar strategy. For $g c v A$, both the forward and reverse primers contained artificial HindIII sites complementary to the $Y$. pestis sequence beginning 111 bases upstream of the gcvA transcription start site and 349 bases downstream of the gcvA translation stop codon. For $g c v R$, both the forward and reverse primers contained artificial HindIII sites complementary to the $Y$. pestis sequence beginning 313 bases upstream of the $g c v R$ transcription start site and 198 bases downstream of the $g c v R$ translation stop codon. The PCR amplified fragments were cloned into the HindIII site of plasmid pACYC177 [24], generating plasmids pgcvA Yp-p177 and p $g c v R^{\mathrm{Yp}}$-p177. In a second construct of $g c v R$, both the upstream and downstream primers contained artificial EcoRI sites and the PCR amplified fragment was cloned into the EcoRI site of plasmid pBR322, generating plasmid $\mathrm{p} g c v R^{\mathrm{Yp}-\mathrm{p} 322}$. Each gene was sequenced at the University of Iowa DNA Core Facility to verify that no bp changes were introduced during the PCR amplification procedure.

\section{Construction of lacZ gene fusions}

Three different transcriptional gene fusions of $g c v B$ to the lacZ gene were constructed by PCR synthesis of fragments with common BamHI termini 128 bp upstream of the $g c v B$ transcription start site and 3 different fusion points

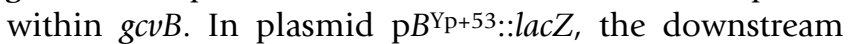
PCR primer hybridized to the $g c v B$ sequence beginning at 
bp +53 relative to the predicted transcription start site $(+1)$ of $g c v B$ (Fig. 1). A synthetic HindIII site was included at the end of the primer to allow the cloning of the 202 bpBamHI-HindIII fragment into the BamHI-HindIII sites of the lacZ transcriptional reporter plasmid pQF50 [25]. Plasmids $\mathrm{p}^{\mathrm{Yp}+164:: l a c Z}$ and $\mathrm{p} B^{\mathrm{Y} p+251}:$ :lacZ were constructed similarly except that the downstream primers used for PCR synthesis hybridized to the $g c v B$ sequence beginning at bp +164 and +251 (Fig. 1), and the 313 and $400 \mathrm{bp}$ fragments produced were cloned into the BamHIHindIII sites of pQF50. Each fusion was sequenced at the University of Iowa DNA Core Facility to verify that the fusions were at the correct sites and that no bp changes were introduced during the PCR amplification procedure. Each $g c v B$ transcriptional fusion was then subcloned into plasmid pMC1403 [26], generating plasmids $\mathrm{p} g c v B^{\mathrm{Yp}+53}:: l a c Z, p g c v B^{\mathrm{Yp}+164:: l a c Z}$ and $\mathrm{p} g c v B^{\mathrm{Yp}+251}::$ lacZ, and subsequently transferred to phage $\lambda g t 2$ [27] as

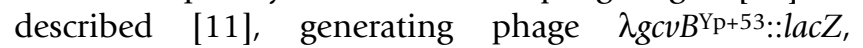

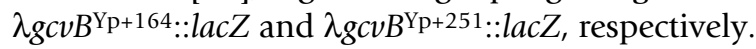

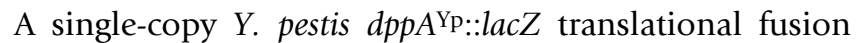

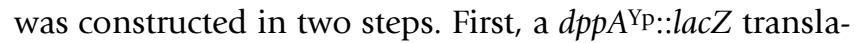
tional fusion was constructed using an upstream PCR primer with an EcoRI site complementary to the Y. pestis DNA sequence beginning 300 bps upstream of the $d p p A$ transcription initiation site and a downstream primer that contains an artificial SmaI site and that hybridizes to the $d p p A$ sequence after the $15^{\text {th }}$ codon relative to the translation initiation site. The $611 \mathrm{bp} d p p A$ fragment was cloned into the EcoRI and SmaI sites of the lacZ translational reporter plasmid pMC1403. The fusion was sequenced at the University of Iowa DNA Core Facility to verify that the fusion was at the correct site and that no bp changes were introduced during the PCR amplification procedure. The $d p p A^{\mathrm{Y}} \mathrm{P}:$ : lac $Z$ fusion, along with the $l a c Y$ and lacA genes, was then cloned into the single-copy plasmid pGS366, designated $\mathrm{p} d p p A^{\mathrm{Yp}:: l a c Z \text {. }}$

\section{Chromosomal deletion of gcvB}

A gcvB deletion was constructed on the Y. pestis chromosome essentially as described [28]. Y. pestis strain KIM6 was transformed with plasmid pKD46, which encodes the Red recombinase of phage $\lambda[28]$. PCR products were then generated using two primers with $50 \mathrm{nt}$ extensions that are complementary to sequences that flank the $g c v B$ gene and $20 \mathrm{nt}$ priming sequences that are complementary to the template plasmid pKD32 and that flank the $\mathrm{CM}^{\mathrm{R}}$ gene and the FLP recognition sequence [28]. The PCR fragment was gel purified and used to transform Y. pestis KIM6 [pKD46]. The cells were plated on HIB plates with $\mathrm{CM}$ and $\mathrm{CM}^{\mathrm{R}}$ recombinants were selected. One $\mathrm{CM}^{\mathrm{R}}$ recombinant was single colony purified, chromosomal DNA was prepared, and PCR analysis was used to verify that the gcvB gene was deleted and replaced with the $\mathrm{CM}^{\mathrm{R}}$ marker. The pKD46 plasmid is a temperature sensitive replicon and was cured by growth at $37^{\circ} \mathrm{C}[28]$. The strain was designated KIM6 $g c v B$.

\section{Authors' contributions}

SM carried out most of the genetic experiments and wrote the first draft of the manuscript. SP carried out the genetic experiments with $g c v R$ and also performed the Northern analysis. GS carried out the computer search to identify putative $g c v B$ genes in other organisms and was the principal investigator and supervised the project.

\section{Acknowledgements}

We are indebted to S. Straley for providing Y. pestis strain KIM6. This work was supported by grant GM069506 from the National Institutes of Health.

\section{References}

I. Perry RD, Fetherston JD: Yersinia pestis--etiologic agent of plague. Clin Microbiol Rev 1997, I O(I):35-66.

2. Deng W, Burland V, Plunkett G, Boutin A, Mayhew GF, Liss P, Perna NT, Rose DJ, Mau B, Zhou S, Schwartz DC, Fetherston JD, Lindler LE, Brubaker RR, Plano GV, Straley SC, McDonough KA, Nilles ML, Matson JS, Blattner FR, Perry RD: Genome sequence of Yersinia pestis KIM. J Bacteriol 2002, I 84( I 6):460 I-46I I.

3. Parkhill J, Wren BW, Thomson NR, Titball RW, Holden MT, Prentice MB, Sebaihia M, James KD, Churcher C, Mungall KL, Baker S, Basham $\mathrm{D}$, Bentley SD, Brooks K, Cerdeno-Tarraga AM, Chillingworth T, Cronin A, Davies RM, Davis P, Dougan G, Feltwell T, Hamlin N, Holroyd S, Jagels K, Karlyshev AV, Leather S, Moule S, Oyston PC, Quail M, Rutherford K, Simmonds M, Skelton J, Stevens K, Whitehead S, Barrell BG: Genome sequence of Yersinia pestis, the causative agent of plague. Nature 200I, 4I3(6855):523-527.

4. Fields KA, Nilles ML, Cowan C, Straley SC: Virulence role of $\mathbf{V}$ antigen of Yersinia pestis at the bacterial surface. Infect Immun 1999, 67( 1 0):5395-5408.

5. Hershberg R, Altuvia S, Margalit H: A survey of small RNA-encoding genes in Escherichia coli. Nucleic Acids Res 2003, 3 I(7): | $813-1820$.

6. Wassarman KM, Repoila F, Rosenow C, Storz G, Gottesman S: Identification of novel small RNAs using comparative genomics and microarrays. Genes Dev 200I, I5(13): I637-I65I.

7. Gottesman S: Micros for microbes: non-coding regulatory RNAs in bacteria. Trends Genet 2005, 21 (7):399-404.

8. Zhang A, Wassarman KM, Ortega J, Steven AC, Storz G: The Smlike Hfq protein increases OxyS RNA interaction with target mRNAs. Mol Cell 2002, 9(I): I I-22.

9. Christiansen JK, Larsen MH, Ingmer H, Sogaard-Andersen L, Kallipolitis $\mathrm{BH}$ : The RNA-binding protein Hfq of Listeria monocytogenes: role in stress tolerance and virulence. J Bacteriol 2004, I 86( I I ):3355-3362.

10. Lenz DH, Mok KC, Lilley BN, Kulkarni RV, Wingreen NS, Bassler BL: The small RNA chaperone Hfq and multiple small RNAs control quorum sensing in Vibrio harveyi and Vibrio cholerae. Cell 2004, I I 8( I):69-82.

II. Urbanowski ML, Stauffer LT, Stauffer GV: The gcvB gene encodes a small untranslated RNA involved in expression of the dipeptide and oligopeptide transport systems in Escherichia coli. Mol Microbiol 2000, 37(4):856-868.

12. Olson ER, Dunyak DS, Jurss LM, Poorman RA: Identification and characterization of dppA, an Escherichia coli gene encoding a periplasmic dipeptide transport protein. J Bacteriol 1991, I 73( I):234-244.

13. Guyer CA, Morgan DG, Staros JV: Binding specificity of the periplasmic oligopeptide-binding protein from Escherichia coli. J Bacteriol 1986, I 68(2):775-779.

14. Manson MD, Blank V, Brade G, Higgins CF: Peptide chemotaxis in E. coli involves the Tap signal transducer and the dipeptide permease. Nature 1986, 32 I(6067):253-256.

15. Mfold algorithm [www.bioinfo.rpi.edu/applications/mfold/
[ww $/ \mathrm{m}$. old/rna] [http://www.bioinfo.rpi.edu/applications/mfold/old/rna] 
16. Miller JH: A short course in bacterial genetics. Cold Spring Harbor Laboratory Press, Cold Spring Harbor, N.Y.; 1992.

17. Ghrist AC, Heil G, Stauffer GV: GcvR interacts with GcvA to inhibit activation of the Escherichia coli glycine cleavage operon. Microbiology 200I, 147(Pt 8):2215-2221.

18. Heil G, Stauffer LT, Stauffer GV: Glycine binds the transcriptional accessory protein GcvR to disrupt a GcvA/GcvR interaction and allow GcvA-mediated activation of the Escherichia coli gcvTHP operon. Microbiology 2002, I48(Pt 7):2203-22I4.

19. Ghrist AC, Stauffer GV: Characterization of the Escherichia coli gcvR gene encoding a negative regulator of gcv expression. J Bacteriol 1995, I 77(17):4980-4984.

20. Vogel HJ, Bonner DM: Acetylornithinase of Escherichia coli: partial purification and some properties. J Biol Chem 1956, 218(1):97-106.

21. Kubota K, Yamamoto A: [Tetanus toxin production. I. Peptone-free medium for the toxin production with special reference to the significance of the bovine heart infusion]. Nippon Saikingaku Zasshi 1966, 2 I (I I ):65I-660.

22. Song YJ, Stinski MF: Effect of the human cytomegalovirus IE86 protein on expression of E2F-responsive genes: a DNA microarray analysis. Proc Natl Acad Sci U S A 2002, 99(5):2836-284I.

23. Bolivar F, Rodriguez RL, Greene PJ, Betlach MC, Heyneker HL, Boyer $\mathrm{HW}$ : Construction and characterization of new cloning vehicles. II. A multipurpose cloning system. Gene 1977, 2(2):95-II3.

24. Chang AC, Cohen SN: Construction and characterization of amplifiable multicopy DNA cloning vehicles derived from the PI5A cryptic miniplasmid. I Bacteriol 1978, |34(3): I |4|-| | 56.

25. Farinha MA, Kropinski AM: Construction of broad-host-range plasmid vectors for easy visible selection and analysis of promoters. J Bacteriol 1990, 172(6):3496-3499.

26. Casadaban MJ, Chou J, Cohen SN: In vitro gene fusions that join an enzymatically active beta-galactosidase segment to amino-terminal fragments of exogenous proteins: Escherichia coli plasmid vectors for the detection and cloning of translational initiation signals. J Bacteriol 1980, I 43(2):97I-980.

27. Panasenko SM, Cameron JR, Davis RW, Lehman IR: Five hundredfold overproduction of DNA ligase after induction of a hybrid lambda lysogen constructed in vitro. Science 1977, 196(4286): |88-|89.

28. Datsenko KA, Wanner BL: One-step inactivation of chromosomal genes in Escherichia coli K-12 using PCR products. Proc Natl Acad Sci U S A 2000, 97( I 2):6640-6645.

29. Wilson RL, Stauffer GV: DNA sequence and characterization of GcvA, a LysR family regulatory protein for the Escherichia coli glycine cleavage enzyme system. J Bacteriol 1994, I 76(10):2862-2868

30. Wilson RL, Urbanowski ML, Stauffer GV: DNA binding sites of the LysR-type regulator GcvA in the gcv and gcvA control regions of Escherichia coli. J Bacteriol 1995, I77(17):4940-4946.

31. Sikkema DJ, Brubaker RR: Outer membrane peptides of Yersinia pestis mediating siderophore-independent assimilation of iron. Biol Met 1989, 2(3): 174-184.
Publish with Biomed Central and every scientist can read your work free of charge

"BioMed Central will be the most significant development for disseminating the results of biomedical research in our lifetime. "

Sir Paul Nurse, Cancer Research UK

Your research papers will be:

- available free of charge to the entire biomedical community

- peer reviewed and published immediately upon acceptance

- cited in PubMed and archived on PubMed Central

- yours - you keep the copyright
BioMedcentral 